\title{
Topical Issue on Ground-Based Facilities (GBF): Results and Experiences from ESA's Ground-Based Facilities Programme in Space Life Sciences
}

\author{
Ruth Hemmersbach ${ }^{1} \cdot$ Jennifer Ngo-Anh $^{2} \cdot$ Martin Zell $^{2}$
}

Published online: 30 April 2016

(C) Springer Science+Business Media Dordrecht 2016

The European Programme for Life and Physical Sciences (ELIPS) of the European Space Agency provides a broad spectrum of unique mission opportunities for Life and Physical Sciences. Since 2009 a relatively small but increasingly important part of ELIPS is the special Ground-Based Facilities (GBF) element which has been providing the scientific user community with the ability to access a large variety of European research laboratories and institutions that simulate some of the conditions that can be found in the space environment. The wide range of research capabilities does not only improve the preparation of space experiments, but can also yield further scientific knowledge of the basic influence of gravity or other space or planetary conditions on life, physical, and interdisciplinary processes in general.

Ruth Hemmersbach

Ruth.Hemmersbach@dlr.de

1 German Aerospace Center, Institute of Aerospace Medicine, Gravitational Biology, Linder Höhe, 51147 Köln, Germany

2 European Space Agency, Human Spaceflight and Operations, Noordwijk, The Netherlands
The advantage of using ground-based facilities has considerably increased the potential of experimenting under simulated space conditions as compared to experiments under real space and microgravity mission opportunities, which at present are still limited. Complementary parameter studies or further post-flight experiments are performed which increase the yield of the flight experiments. This efficient approach allows for more comprehensive research programmes, linking them closely to Earth-based research activities and favouring the development of a strong community of scientists from all research disciplines who have the ambition to include challenging objectives of space-relevant research into their daily scientific activities on Earth.

This topical issue demonstrates the success of the GBF programme to be a highly valuable low-cost tool for scientists to push forward their understanding of basic science aspects, preparing very efficiently for more complex spaceflight experiments and advancing important human and biological aspects for exploration. Unfortunately, the GBF programme is currently on hold due to funding shortages in ELIPS - 4 and scientists hope for its continuation in future. To point this out, they contributed in short-term and high effort to the finalisation of this topical issue. The editors like to thank the authors for their contributions and their efficient collaboration. 